\title{
Room temperature test of the continuous spontaneous localization model using a levitated micro-oscillator
}

\author{
Di Zheng, ${ }^{1}$ Yingchun Leng, ${ }^{1}$ Xi Kong, ${ }^{1}$ Rui Li, ${ }^{2,3,4}$ Zizhe Wang, ${ }^{2,3,4}$ Xiaohui Luo, ${ }^{1}$ Jie Zhao, ${ }^{2,3,4}$ Chang-Kui Duan, ${ }^{2,3,4}$

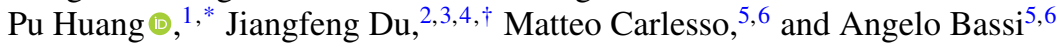 \\ ${ }^{1}$ National Laboratory of Solid State Microstructures and Department of Physics, Nanjing University, Nanjing 210093, China \\ ${ }^{2}$ Hefei National Laboratory for Physical Sciences at the Microscale and Department of Modern Physics, \\ University of Science and Technology of China, Hefei 230026, China \\ ${ }^{3}$ CAS Key Laboratory of Microscale Magnetic Resonance, University of Science and Technology of China, Hefei 230026, China \\ ${ }^{4}$ Synergetic Innovation Center of Quantum Information and Quantum Physics, \\ University of Science and Technology of China, Hefei 230026, China \\ ${ }^{5}$ Department of Physics, University of Trieste, Strada Costiera 11, 34151 Trieste, Italy \\ ${ }^{6}$ Istituto Nazionale di Fisica Nucleare, Trieste Section, Via Valerio 2, 34127 Trieste, Italy
}

(Received 8 June 2019; published 17 January 2020)

\begin{abstract}
The continuous spontaneous localization (CSL) model predicts a tiny break of energy conservation via a weak stochastic force acting on physical systems, which triggers the collapse of the wave function. Mechanical oscillators are a natural way to test such a force; in particular, a levitated micromechanical oscillator has been recently proposed to be an ideal system. We report a proof-of-principle experiment with a micro-oscillator generated by a microsphere diamagnetically levitated in a magnetogravitational trap under high vacuum. Due to the ultralow mechanical dissipation, the oscillator provides a new upper bound on the CSL collapse rate, which gives an improvement of two orders of magnitude over the previous bounds in the same frequency range, and partially reaches the enhanced collapse rate suggested by Adler. Although being performed at room temperature, our experiment has already exhibited advantages over those operating at low temperatures. Our results experimentally show the potential for a magnetogravitational levitated mechanical oscillator as a promising method for testing the collapse model. Further improvements in cryogenic experiments are discussed.
\end{abstract}

DOI: 10.1103/PhysRevResearch.2.013057

\section{INTRODUCTION}

The perceived absence of macroscopic quantum superposition has attracted the physicists' interests since the birth of quantum mechanics. Different interpretations and reformulations of quantum mechanics [1-7] have been proposed to comprehensively handle such an issue, however, most of them do not provide direct experimental testability.

A phenomenological and experimentally verifiable [8-15] approach is proposed by collapse models [16]. They introduce nonlinear and stochastic terms in the Schrödinger equation, which induce a spontaneous collapse of the wave function. Such a collapse is stronger the larger is the system. The origin of the noise remains an open question, and often in the literature it has been linked to gravity [17-19]. In this paper, we focus on the continuous spontaneous localization (CSL) model, one of the most studied in the literature.

\footnotetext{
*hp@nju.edu.cn

$\dagger$ djf@ustc.edu.cn
}

Published by the American Physical Society under the terms of the Creative Commons Attribution 4.0 International license. Further distribution of this work must maintain attribution to the author(s) and the published article's title, journal citation, and DOI.
CSL is characterized by two phenomenological parameters: the collapse rate $\lambda$ and its correlation length $r_{C}$. The latter can be also understood as the minimum superposition separation necessary to trigger a collapse. The theoretically suggested values for these parameters are $\lambda \simeq 10^{-16} \mathrm{~s}^{-1}$ and $r_{\mathrm{C}}=10^{-7} \mathrm{~m}$ by Ghirardi et al. [8,10], while larger values were considered by Adler, $\lambda \simeq 10^{-8 \pm 2} \mathrm{~s}^{-1}$ at $r_{\mathrm{C}}=10^{-7} \mathrm{~m}$, and $\lambda \simeq 10^{-6 \pm 2} \mathrm{~s}^{-1}$ at $r_{\mathrm{C}}=10^{-6} \mathrm{~m} \mathrm{[20] \text {. }}$

The CSL modifications to standard quantum mechanics cause primarily a loss of coherence, which has been studied using cold atoms [21], molecular interferometers [22-27], and phonon motion in diamonds [28,29]. These compose the first class of experiments, namely, the interferometric ones. Collapse models also predict a stochastic force noise acting on the system, whether it is in a quantum or classical state. This opens the way for noninterferometric experiments [11,12,15], which have already constrained significantly the parameter space of the CSL model. To this class belong experiments with ultracold atoms [30,31], bulk solid matter [32-34], planetary temperature observations [35-37], spontaneous x-ray emissions [38-40], and optomechanical systems [41-49]. Many more proposals were analyzed [50-57].

Noninterferometric experiments test the collapse mechanism at different frequencies, ranging from $\mathrm{mHz}[43,44]$ to $10^{19} \mathrm{~Hz}$ [39]. Since the CSL noise is originally assumed to be white, the bound on the collapse parameters is 
independent from the frequency at which the collapse mechanism is probed. However, this is not the case for colored extensions of the model, where the noise is no longer white and is typically characterized by a frequency cutoff [20,58-60].

Recently, several studies $[26,31,33,61]$ were performed in this direction, indicating the urgency of testing the CSL noise at different frequencies to probe its spectrum. Optomechanics provides the optimal platform for this scope, since frequencies range from sub-mHz to $\mathrm{kHz}$ or even higher [62]. Among them, the gravitational wave detectors AURIGA, Advanced LIGO, and LISA Pathfinder, due to their large test mass, succeeded in setting strong bounds on collapse parameters $[45,46,51,63]$ at frequencies less than $1 \mathrm{kHz}$ [41], tens of $\mathrm{Hz}$ [42], and sub- $\mathrm{Hz}$ $[43,44]$, respectively. Among them, LISA Pathfinder gives the strongest upper bound on $\lambda[44,51]$. Also, microscale solidstate force sensors such as nanocantilevers provided precise testing of the collapse noise $[47,48]$ at frequencies above $\mathrm{kHz}$. In this case, the relative large damping rates are balanced by operating at millikelvin temperatures.

Levitated micro- or nanomechanical oscillators are ideal for potentially testing collapse models due to their low damping rates. Although they recently attracted considerable theoretical interest [52,63-66], an experimental demonstration of their ability for such a purpose has not yet been performed.

Here, we report a proof-of-principle test of CSL based on a magnetically levitated micromechanical oscillator at room temperature. The levitation is realized with a specially designed magnetogravitational trap where a test particle of mass of $4.7 \mathrm{pg}\left(\sim 2.8 \times 10^{12} \mathrm{amu}\right)$ is stably levitated for some days in high vacuum. We observed a damping rate $\gamma / 2 \pi$ of the order of $30 \mu \mathrm{Hz}$ at a resonant frequency of the order of $10 \mathrm{~Hz}$. This underlines the noiseless character of magnetogravitational traps, that can actually provide a sensitive instrument for collapse model testing. As we will discuss below, for $r_{\mathrm{C}}=10^{-7} \mathrm{~m}$, we estimate the upper bound $\lambda=10^{-6.4} \mathrm{~s}^{-1}$ on the collapse rate at $95 \%$ confidence level, excluding part of the range of values of the CSL parameters suggested by Adler [20]. This is a significant improvement with respect to the bound obtained from the gravitational-wave detector Advanced LIGO which operates at the same frequency range $[42,45]$ and proves that magnetogravitational levitation is a strong competitive platform for testing the limits of quantum mechanics.

\section{THEORETICAL MODEL}

According to the mass-proportional CSL model [11], the collapse of the wave function leads to a spontaneous diffusion process, which is described by the Lindblad term [13-15],

$$
\mathcal{L}_{\mathrm{CSL}}\left[\hat{\rho}_{s}(t)\right]=-\eta_{i}\left[\hat{x}_{i},\left[\hat{x}_{i}, \hat{\rho}_{s}(t)\right]\right],
$$

where $\hat{\rho}_{s}(t)$ is the density operator describing the center of mass motion, $i=x, y, z$ labels the direction of motion, and

$$
\eta_{i}=\frac{r_{\mathrm{C}}^{3} \lambda}{\pi^{\frac{3}{2}} m_{0}^{2}} \int d^{3} \mathbf{k}\left|\tilde{\mu}_{s}(\mathbf{k})\right|^{2} k_{i}^{2} e^{-k^{2} r_{\mathrm{C}}^{2}}
$$

is the CSL diffusion constant, which depends on the geometry of the object. Here, $m_{0}$ is the atomic mass unit, $k=|\mathbf{k}|$ with $\mathbf{k}=\left(k_{x}, k_{y}, k_{z}\right)$, and $\tilde{\mu}_{s}(\mathbf{k})$ is the Fourier transform of the mass density $\mu_{s}(\mathbf{r})$, i.e., $\tilde{\mu}_{s}(\mathbf{k})=\int d \mathbf{r}^{3} e^{i \mathbf{k} \cdot \mathbf{r}} \mu_{s}(\mathbf{r})$. In this experiment, the system is a sphere of radius $R$ and mass $m$, for which we have $\tilde{\mu}_{s}(\mathbf{k})=3 m[\sin (k R)-k R \cos (k R)] /(k R)^{3}$. By substituting $\tilde{\mu}_{s}(\mathbf{k})$, we obtain a single diffusion constant independently from the direction of motion,

$$
\eta=\frac{6 r_{\mathrm{C}}^{4} \lambda m^{2}}{m_{0}^{2} R^{6}}\left[e^{-R^{2} / r_{\mathrm{C}}^{2}}-1+\frac{R^{2}}{2 r_{\mathrm{C}}^{2}}\left(e^{-R^{2} / r_{\mathrm{C}}^{2}}+1\right)\right] .
$$

The CSL-induced center of mass diffusion can be effectively described by a stochastic force $f_{\mathrm{CSL}}(t)$ with zero mean and correlation $\left\langle f_{\mathrm{CSL}}(t) f_{\mathrm{CSL}}(s)\right\rangle=S_{\mathrm{CSL}} \delta(t-s)$, where $S_{\mathrm{CSL}}=$ $\hbar^{2} \eta$ is the CSL force spectral density.

We describe the dynamics of our mechanical system as a damped harmonic oscillator subject to environmental and, potentially, CSL noises. Dropping the label $i$, the motion in one direction of the center of mass of our system reads

$$
m \ddot{x}+m \gamma \dot{x}+k x=f_{\mathrm{th}}(t)+f_{\mathrm{CSL}}(t),
$$

with $\gamma / 2 \pi$ the damping rate, and $k=m \omega_{0}^{2}$ the spring constant. The first term on the right-hand side represents the thermal Brownian force noise, whose correlations read $\left\langle f_{\mathrm{th}}(t) f_{\mathrm{th}}(s)\right\rangle=S_{\mathrm{th}} \delta(t-s)$, where $S_{\mathrm{th}}=2 \gamma m k_{\mathrm{B}} T_{\mathrm{env}}$ the corresponding power spectral density, which is fully characterized by the environmental temperature $T_{\text {env }}$ [67]. For a system in thermal equilibrium, the additional presence of the collapse force $f_{\mathrm{CSL}}(t)$ leads to an increase of the temperature of the system [15]. The effective temperature is thus $T_{\mathrm{eff}}=T_{\mathrm{env}}+$ $T_{\mathrm{CSL}}$, where $T_{\mathrm{CSL}}$, the CSL induced temperature contribution, satisfies the relation $2 \gamma m k_{\mathrm{B}} T_{\mathrm{CSL}}=\hbar^{2} \eta$. Here, one assumes that $f_{\text {th }}(t)$ and $f_{\mathrm{CSL}}(t)$ are independent. Figure 1(a) shows an intuitive picture of the thermal equilibrium dynamics of the magnetogravitationally levitated microsphere used in this experiment.

It is clear that any other source of noise, such as, for example, that due to the measurement back-action, also contributes to the total noise [68]. In Appendix A we discuss different possible noise processes involved in the experiment; however, we take a conservative approach and consider all nonthermal noise as potential CSL noise in setting the upper bound.

The total power spectral density is defined as $S_{\text {total }}=$ $2 \gamma m k_{\mathrm{B}} T_{\text {eff }}$, which is calculated from the measured $T_{\text {eff }}$. By subtracting the thermal Brownian contribution $S_{\text {th }}$, we obtain the power spectral density of all additional force noises $\delta S_{\text {total }}=S_{\text {total }}-S_{\text {th }}$. Therefore, $\delta S_{\text {total }}=2 \gamma m k_{\mathrm{B}} \delta T$ provides the estimation of the upper bound of the CSL force as $\hbar^{2} \eta \leqslant$ $\delta S_{\text {total }}$, with $\delta T=T_{\text {eff }}-T_{\text {env }}$ denoting the rise of the effective temperature. Note that, apart from a barely tunable material density $\sim m / R^{3}$ [cf. Eq. (3)], the ability to test CSL is limited only by the accuracy in determining the thermal Brownian noise. Different methods for such noise signal sensing have been developed for similar purposes [68-71].

\section{EXPERIMENT DESCRIPTION AND RESULTS}

The levitation of diamagnetic systems using magnetogravitational forces has been already performed with either a superconductor [72] or permanent magnets [73]. The magnetogravitational trap used in our experiment was generated by a set of micromachined $\mathrm{NdFeB}$ magnets with octagonal bilayer geometry as shown in Fig. 1(b). In Appendix B, 
(a)

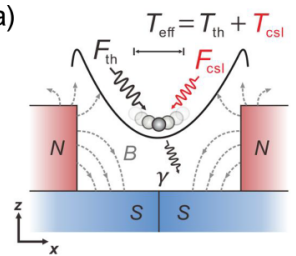

(c)

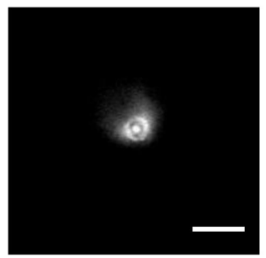

(b)

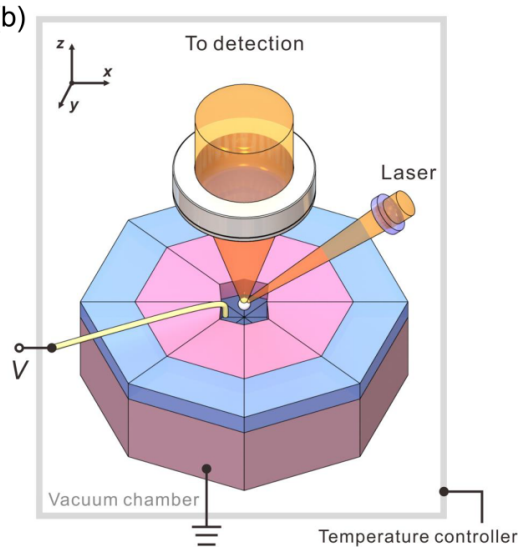

FIG. 1. Basic concept of the experiment and setup. (a) Scheme of the oscillator dynamics. A microsphere trapped in a magnetogravitational potential (black curve) is subject to Brownian motion with an effective temperature $T_{\text {eff }}$. In absence of the CSL collapse force $f_{\mathrm{CSL}}$, the thermal Brownian force $f_{\text {th }}$ leads to an effective $T_{\text {eff }}$ which is equal to the environment temperature $T_{\text {env }}$. When $f_{\mathrm{CSL}}$ is added, the effective temperature will rise by $T_{\mathrm{CSL}}$. The smaller the oscillator damping rate $\gamma / 2 \pi$, the higher is the effective temperature. (b) Experiment setup. A diamagnetic microsphere is levitated in a magnetogravitational trap generated by a set of permanent magnets (red and blue indicating the $\mathrm{N}$ and $\mathrm{S}$ poles-see Appendix B for details). A laser is focused on the microsphere and an objective is used to collect the scattered light. An electrode is placed near the microsphere and an electric field is applied to determine the charge state of the microsphere. The whole setup is placed in a vacuum chamber, whose temperature is controlled (see Appendix $\mathrm{B}$ for details). (c) Optical image of the microsphere. The microsphere used in our experiment with a radius of $1 \mu \mathrm{m}$ (the scale bar being $5 \mu \mathrm{m}$ ).

we report details about the trap design. The oscillator is a microsphere of polyethylene glycol, whose magnetic susceptibility is $-9.1 \times 10^{-6}$ and its density is $1.1 \times 10^{3} \mathrm{~kg} / \mathrm{m}^{3}$. The microsphere is generated using a home-built nebulizer. A 633-nm laser is focused on the droplet with a power less than $50 \mu \mathrm{W}$, and the scattered light from the microsphere is collected with an objective. The position of the microsphere is tracked with a CCD camera, and its motion is recorded in the time domain with a photon detector. To isolate the trap from external vibrations, the trap is mounted on a heavy copper frame, which is suspended in a vacuum chamber by means of springs. Because the environmental temperature fluctuations contribute to the measurement uncertainty of the effective temperature of the oscillator, a double layer vacuum chamber and a proportional-integral-derivative (PID) temperature controller are used to maintain a stable environmental temperature. In this way, we achieved fluctuations smaller than $100 \mathrm{mK}$ with an environmental temperature set to $298 \mathrm{~K}$ over the whole duration of the experiment $\left(\sim 10^{5}-10^{6} \mathrm{~s}\right)$.

We observed that for electrically charged particles, the dissipation in the experiment is higher than with no charge. This can lead to a strong instability of the particles' motion, which makes them eventually escape from the trap. To avoid this, the charge on the microsphere is eliminated by using ultraviolet light. Subsequently, the charge state is

checked via a microelectrode made of a $40-\mu \mathrm{m}$-diam gold wire placed near the trapping center. By applying a voltage $\sim 50 \mathrm{~V}$, microspheres with a radius of less than $2 \mu \mathrm{m}$ can be easily pulled out of the trap if the net charge is nonzero (see Appendix $\mathrm{C}$ for further details). Even after having removed the charges, at room temperature, microspheres with a radius smaller than $500 \mathrm{~nm}$ are found to escape the trap due to the thermal fluctuation and the limited depth of the trap (see Appendix B for details). The remaining particles were left in the trap in high vacuum for several days. If the particle did not evaporate during this time, it eventually reached the equilibrium thermal distribution, which was observed to be stable within the measurement error.

For microspheres of radius close to $1 \mu \mathrm{m}$, the direct optical image failed to provide a reliable estimation of the size of the system, and we determined it through the following two methods. In the first method, we made use of the relation between the microsphere oscillation damping rate due to the background gas collisions and the pressure, which reads $\gamma=(16 / \pi)(P / \nu R \rho)$ (holding for high pressures) [74], with $P$ and $v$ the pressure and the mean speed of the background gas, respectively. In this part of the experiment, the pressure was set to $P \sim 10^{-3}$ mbar, so that the damping was fully dominated by the background gas; $v$ can be inferred from the environmental temperature, and by measuring $\gamma$ one obtains an estimate for $R$.

The second method simply relied on the equipartition theorem. We measured the oscillator displacement distribution, which follows a Gaussian distribution $P(x) \propto \exp \left(-x^{2} / 2 \sigma^{2}\right)$, thus determining its standard deviation $\sigma$. The latter is related to the size of the particle through the energy equipartition theorem, $4 \pi \sigma^{2} \mu R^{3} \omega_{0}^{2}=3 k_{\mathrm{B}} T_{\mathrm{env}}$, with $\omega_{0}$ the resonant frequency of the oscillator. The results from the two methods are compatible. The microsphere used in the experiments, whose results are described below, has radius $R=1.0 \mu \mathrm{m}$, corresponding to $4.7 \mathrm{pg}$ and with a corresponding potential energy which is thousands of times larger than the thermal energy $k_{\mathrm{B}} T$.

After successfully capturing the microsphere and eliminating its charge, we proceeded to measure the effective temperature $T_{\text {eff }}$ associated with the center of mass motion of the particle. As a first step, we set a medium vacuum $\left(P_{\mathrm{MV}} \sim 10^{-4}\right.$ mbar $)$, and measured the position distribution of the microsphere in the $x-y$ plane. A typical example of the measured data in a run of few minutes is plotted in Fig. 2. The distribution has an elliptical shape due to the asymmetry of the trap. The distribution can be fitted with a two-dimensional Gaussian distribution, whose long (axis 1) and short (axis 2) standard deviations are denoted by $\sigma_{1}$ and $\sigma_{2}$, respectively. The energy equipartition theorem, which implies $\sigma_{1} / \sigma_{2}=\omega_{1} / \omega_{2}$, is well satisfied within the measurement error, where $\omega_{1}=12.9 \mathrm{~Hz}$ and $\omega_{2}=9.3 \mathrm{~Hz}$ are the corresponding resonance frequencies (see Appendix D for details on the displacement power spectral density). The effective temperature is then calculated as $T_{\text {eff }}=m \omega_{1}^{2} \sigma_{1}^{2} / k_{\mathrm{B}}$ (equally, $m \omega_{2}^{2} \sigma_{2}^{2} / k_{\mathrm{B}}$ ). Since at a medium vacuum, the thermal Brownian noise from the background gas fully dominates the other noise, we assume that $S_{\text {total }}=S_{\text {th }}$ and we use this relation to calibrate the environmental temperature as $T_{\text {env }}=T_{\text {eff }}$. Without loss of generality, we make 


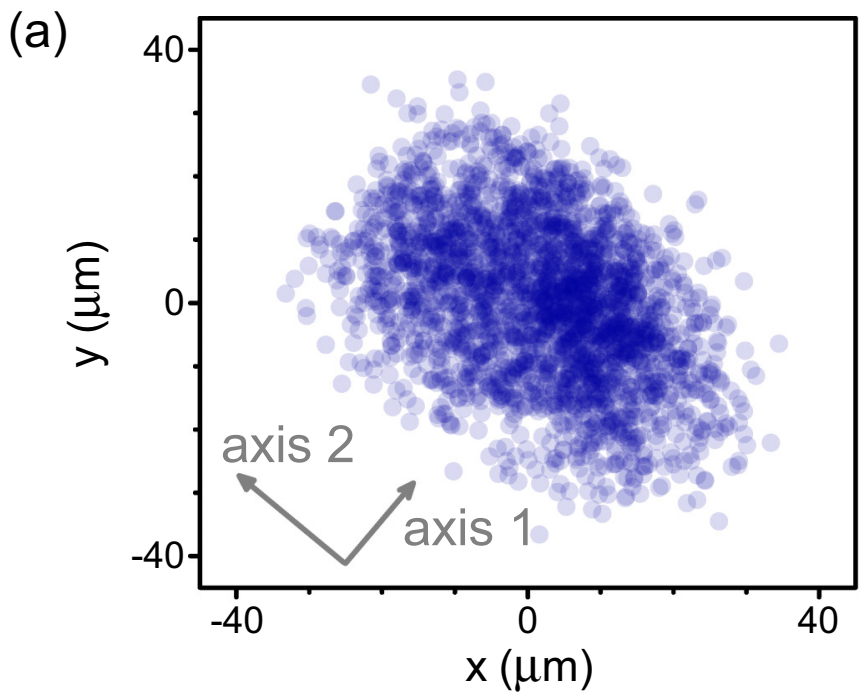

(b)

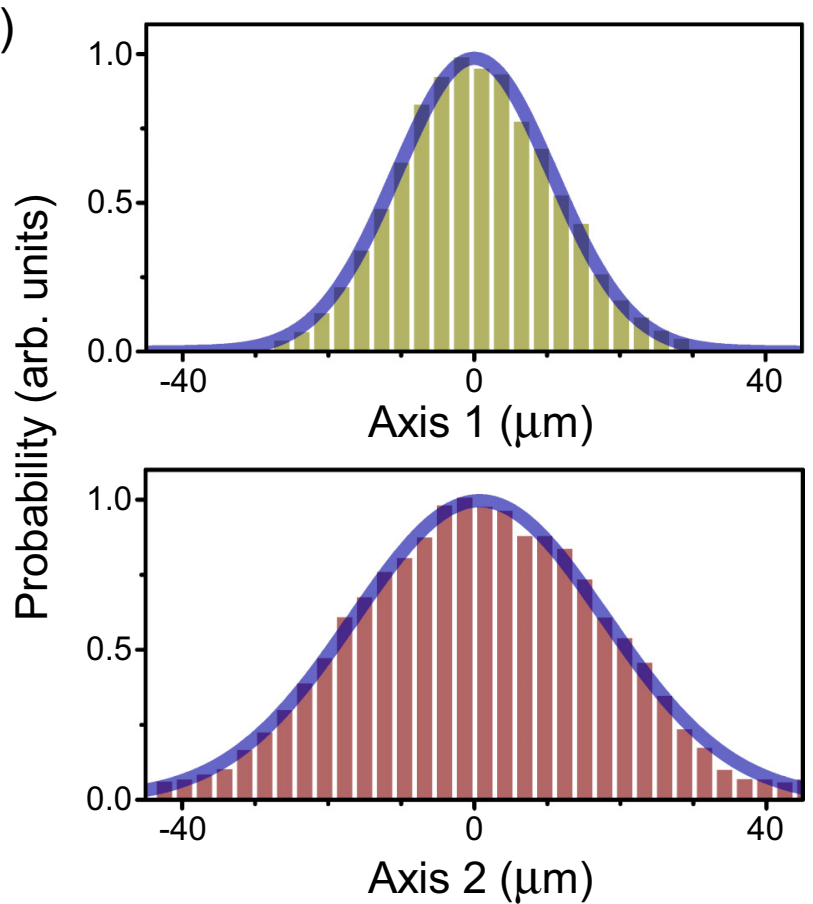

FIG. 2. Measurements of the distribution in position of the microsphere. (a) Typical position distribution in the $x-y$ plane. The data were measured under medium vacuum for a few minutes. Axis 1 (short) and axis 2 (long) of the distribution correspond to the modes with resonance frequencies $\omega_{1}=12.9 \mathrm{~Hz}$ and $\omega_{2}=9.3 \mathrm{~Hz}$, respectively. (b) and (c) Displacement distributions along axis 1 and axis 2, respectively. Data are fitted with a Gaussian distribution (blue curve), from which the effective temperature is determined.

use of mode $1\left(\omega_{1} / 2 \pi \approx 12.9 \mathrm{~Hz}\right)$ for the subsequent measurement.

Next, we determine the dissipation constant $\gamma$, which is another key factor for determining the thermal Bronwnian force noise strength. First, we note that, for $P<10^{-5} \mathrm{mbar}$, the measured power spectral density $S_{x}(\omega)$ shows a strong asymmetric character, deviating substantially from a Lorentzian shape, and is considerably broadened compared to that estimated from the background gas. Such a feature is known
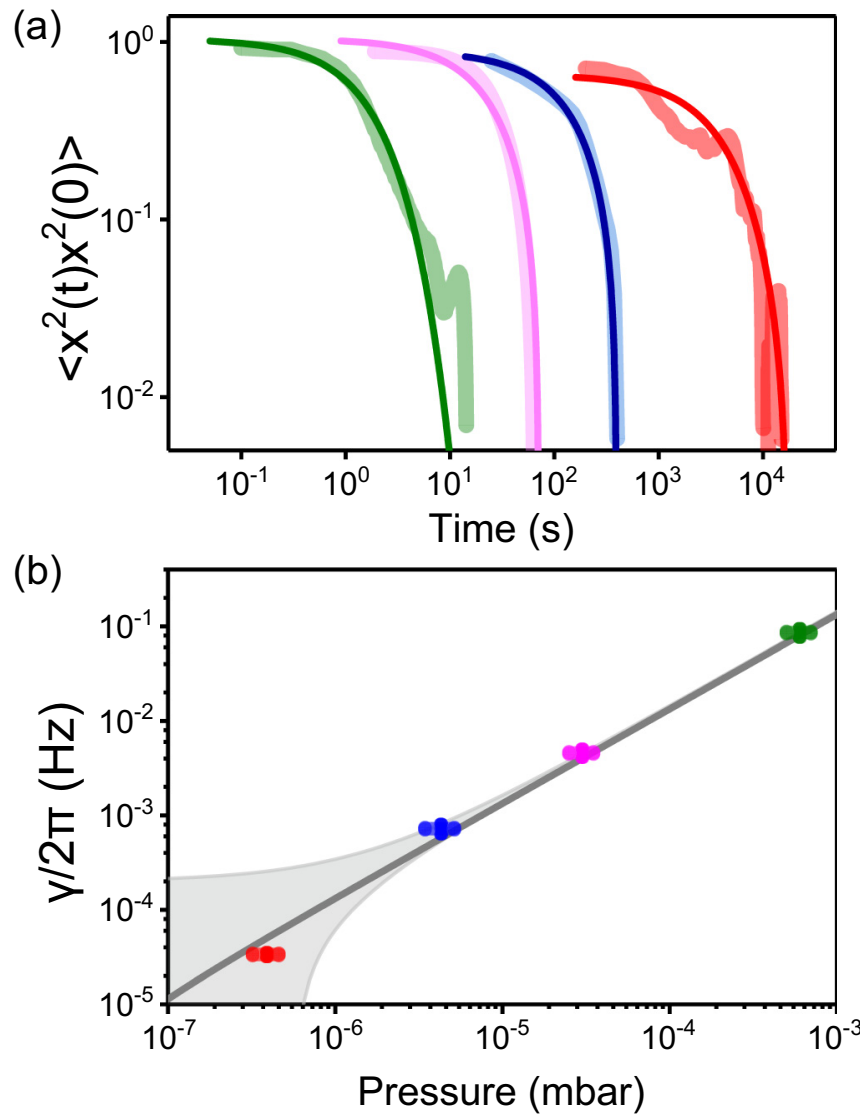

FIG. 3. Measurement of the damping rate. (a) Autocorrelation functions of the oscillator energy measured at the pressures given in (b). Data are fitted with an exponential decaying function $\exp (-t / \tau)$. The decay time $\tau$ is determined for each curve by the best fit. A total averaged time, typically 50 times longer than $\tau$, was carried out for each curve to achieve a good signal-to-noise ratio. (b) Dependence of the damping rate $\gamma / 2 \pi=1 /(2 \pi \tau)$ on the pressure, where $\tau$ is obtained from (a). The gray line is a linear fit, where the vertical and horizontal error bars are due to a fitting error and the pressure imprecision of the vacuum chamber, respectively. The shaded gray area corresponds to the $95 \%$ confidence band.

[75] and is due to the nonlinearity of the trap. Therefore, to estimate $\gamma$, we follow the prescription of Ref. [76] and make use of the energy autocorrelation defined as $\left\langle X^{2}(t) X^{2}(0)\right\rangle$, with $X(t)$ the amplitude of the oscillation. This method is insensitive to the nonlinearity of the trap (see Appendix D for details). The measured autocorrelation curve is then fitted to the exponentially decaying function $\exp (-t / \tau)$, from which we obtain the damping rate $\gamma=1 / \tau$. Figure 3(a) shows the measured energy autocorrelation for different values of the pressure. In particular, at the highest vacuum $P_{\mathrm{HV}} \approx 4 \times$ $10^{-7}$ mbar, the measured decay time $\tau \approx 4700$ s corresponds to a damping rate $\gamma / 2 \pi \approx 34 \mu \mathrm{Hz}$. We also find that the damping rate decreases linearly as the pressure decreases, which shows that the background gas remains the dominant dissipative channel in the experiment, as shown in Fig. 3(b). Combining the measured effective damping rate $\gamma / 2 \pi$ and temperature $T_{\text {eff }}$, we estimate a force sensitivity of the oscillator in high vacuum as $\sqrt{S_{\text {total }}}=9.6 \times 10^{-20} \mathrm{~N} / \sqrt{\mathrm{Hz}}$. This 
TABLE I. Upper bounds on the CSL collapse rate $\lambda$. $\delta T$ is defined as the temperature increase $\delta T=T_{\text {eff }}-T_{\text {env }}$, with $T_{\text {eff }}$ and $T_{\text {env }}$ the effective temperature of the oscillator measured at high vacuum and the environment temperature, respectively. $\sqrt{\delta S_{\text {total }}}$ is the measured additional force noise beyond the thermal force. $\sigma_{\delta T}$ and $\sigma_{\sqrt{\delta S_{\text {total }}}}$ are the corresponding standard deviations at 95\% confidence level. Finally, the upper bounds on $\lambda$ at $95 \%$ confidence level are the calculated for $r_{\mathrm{C}}=10^{-7} \mathrm{~m}$ and $r_{\mathrm{C}}=10^{-6} \mathrm{~m}$.

\begin{tabular}{|c|c|c|c|c|c|}
\hline \multicolumn{2}{|c|}{ Excess temperature } & \multicolumn{2}{|c|}{ Excess noise } & \multicolumn{2}{|c|}{ Upper bound on the collapse rate } \\
\hline$\delta T$ & $\sigma_{\delta T}$ & $\sqrt{\delta S_{\text {total }}}$ & $\sigma_{\sqrt{\delta S_{\text {total }}}}$ & $\lambda\left(r_{\mathrm{C}}=10^{-7} \mathrm{~m}\right)$ & $\lambda\left(r_{\mathrm{C}}=10^{-6} \mathrm{~m}\right)$ \\
\hline $6.5 \mathrm{~K}$ & $40 \mathrm{~K}$ & $1.3 \times 10^{-20} \mathrm{~N} / \sqrt{\mathrm{Hz}}$ & $3.3 \times 10^{-20} \mathrm{~N} / \sqrt{\mathrm{Hz}}$ & $10^{-6.4} \mathrm{~s}^{-1}$ & $10^{-7.4} \mathrm{~s}^{-1}$ \\
\hline
\end{tabular}

value is comparable to that obtained from optical trapping [77].

By comparing the power spectral densities at medium and high vacuum we find the upper bound on the collapse rate $\lambda$; the main results are summarized in Table I. For medium vacuum, the background gas is coupled to the system, thus maintaining the temperature of the system at equilibrium with the environmental one. On the contrary, in the high vacuum condition, the gas decouples, and thus any potential CSL contribution is not dissipated, thus imposing an effective temperature which is higher than $T_{\text {env }}$. To bound the CSL effect we proceed as follows. The power spectral density of nonthermal forces is estimated via $\delta S_{\text {total }}=2 m \gamma k_{\mathrm{B}} \delta T$, where $\gamma$ is measured at high vacuum, $\delta T=T_{\text {eff }}-T_{\text {env }}$, with $T_{\text {eff }}$ being calculated from the standard deviation of the position distribution $\sigma$ at high vacuum and with $T_{\text {env }}$ measured at medium vacuum. We obtain the upper bound $\sqrt{\delta S_{\text {total }}}<3.3 \times$ $10^{-20} \mathrm{~N} / \sqrt{\mathrm{Hz}}$ at the $95 \%$ confidence level (see Appendix E for details about measurements of $T_{\text {eff }}$ and error estimation). Accordingly, the bound on $\lambda$ is calculated through Eq. (3).

Figure 4(a) compares the excluded values of $\lambda$ at different frequencies for $r_{\mathrm{C}}=10^{-7} \mathrm{~m}$. In particular, our experiment improves by more than two orders of magnitude the bound posed by Advanced LIGO [42,45] at the same frequency.

The upper bound provided by this experiment also partially excludes the range of values of collapse rate suggested by Adler for $r_{\mathrm{C}}=10^{-7} \mathrm{~m}$ [20], and almost entirely excludes it for $r_{\mathrm{C}}=10^{-6} \mathrm{~m}$. We also estimated the performances of this experiment by using parameters that are more favorable for CSL testing and which are potentially achievable with our experiment by working at cryogenic condition and for $10^{-8}$ mbar pressure: $R=0.3 \mu \mathrm{m}, \gamma / 2 \pi=10^{-6} \mathrm{~Hz}$, and $\delta T=10 \mathrm{mK}$. A negative result would imply $\lambda \leqslant 10^{-11.9} \mathrm{~s}^{-1}$ for $r_{\mathrm{C}}=10^{-7} \mathrm{~m}$, which would fully rule out Adler's suggestion. The comparison of our experimental upper bound and the hypothetical upper bound with the strongest bounds reported in the literature are shown in Fig. 4(b), together with the theoretical values for the collapse parameters.

\section{SUMMARY AND DISCUSSION}

Levitated oscillators have been recently proposed as suitable systems for collapse model testing [52,63-66]. Here, we demonstrated that an experiment based on a magnetogravitational levitated micro-oscillator can place important bounds on the collapse parameters although operating at room temperature. We obtained a new upper bound, which is a significant improvement over previous results in the same frequency range and it partially probes Adler's theoretical proposal. The system reported here shows a great potential, which would be fully expressed at cryogenic temperatures, where an improvement of several orders of magnitude in bounding the collapse noise is expected.

The performance of the current experiment at room temperature is mainly limited by three factors, which eventually could be improved significantly at lower temperatures. First, the effective temperature measurement precision is worse than tens of degrees Kelvin but is expected to reach $\mathrm{mK}$ under cryogenic conditions. Differently from other kinds of levitated micro-oscillators, such as electrical [78], optical [74,77,79], and magnetic levitation [80-82], our magnetogravitational trap is fully passive with no energy inputs. Thus, it is naturally suitable for low temperature conditions. (In principle, lasers generate an addition force noise. However, the laser intensity is weak at room temperature. Its impact at cryogenic temperatures is still to be evaluated.) Second, the minimum radius of the microsphere in this experiment is currently limited by the thermal energy, thus, at low temperature, a much smaller microsphere could be stably trapped and lead to higher precision in detection. The third potential of improvement is dissipation, which is observed to be constrained by the pressure. Room temperature experiments show that a higher vacuum does not lead to a significantly improvement in dissipation [73], since eventually other dissipative channels will contribute at lower pressures. However, it is yet to be explored whether dissipation can decrease at a much lower temperature environment. This work opens a new door for the precise study of collapse models and may provide promising avenues towards breakthrough discoveries in the future.

Note added in proof. Recently, we became aware of similar independent work by Pontin et al. [49].

\section{ACKNOWLEDGMENTS}

We thank L. Diósi for helpful comments. We thank Xuan He, Zhangqi Yin, Zhujing Xu, Jingwei Zhou, Fei Xue, and Xing Rong for helpful discussions. This work was supported by the National Key R\&D Program of China (Grant No. 2018YFA0306600), the National Natural Science Foundation of China (Grants No. 61635012, No. 11675163, No. 11890702, No. 81788101, No. 11761131011, and No. 11722544), the CAS (Grants No. QYZDY-SSW-SLH004 and No. GJJSTD20170001), the Fundamental Research Funds for the Central Universities (Grant No. 021314380149), and the Anhui Initiative in Quantum Information Technologies (Grant No. AHY050000). M.C. and A.B. acknowledge financial support from the H2020 FET Project TEQ (Grant No. 766900). A.B. acknowledges financial support from the COST Action QTSpace (CA15220), INFN, and the University of Trieste. 


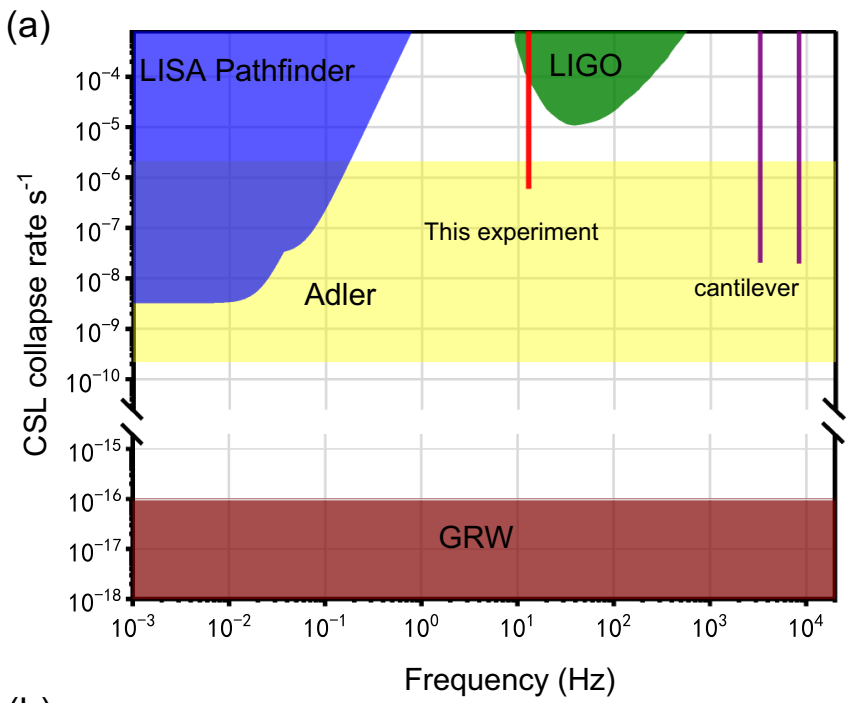

(b)

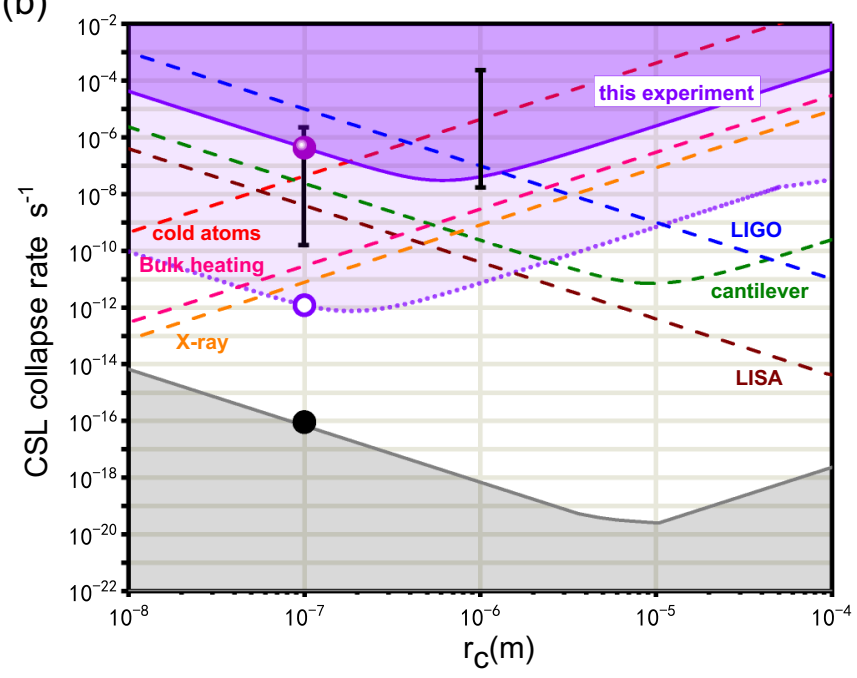

FIG. 4. Exclusion plot of the CSL parameters. (a) Upper bounds on the CSL collapse rate $\lambda$ from mechanical-based experiments, for $r_{\mathrm{C}}=10^{-7} \mathrm{~m}$. Red line: Upper bound given by our experiment at the $95 \%$ confidence level. Blue and green regions, and purple lines: Exclusion regions obtained from LISA Pathfinder [43-46,51], Advanced LIGO [42,45], and millikelvin cantilever experiments $[47,48]$, respectively. The yellow region is the range proposed by Adler for $\lambda$ [20]. The claret region is the value proposed by Ghirardi, Rimini, and Weber (GRW) [8] and it works as a lower theoretical bound. (b) Upper bounds in the $\lambda-r_{\mathrm{C}}$ plane given by our experiment, compared with the best experimental upper bounds reported so far as well as proposed theoretical lower bounds. The purple solid line and corresponding shaded region: Upper bound and exclusion region given by our experiment. Purple dotted line: Upper bound estimated with parameters $R=0.3 \mu \mathrm{m}, \gamma / 2 \pi=10^{-6} \mathrm{~Hz}$, and $\delta T=10 \mathrm{mK}$. At $r_{\mathrm{C}}=10^{-7} \mathrm{~m}$, values of the collapse rate $\lambda$ obtained by this experiment and its future possible improvement are marked by a purple solid $\operatorname{dot}\left(\lambda=10^{-6.4} \mathrm{~s}^{-1}\right)$ and a purple open $\operatorname{dot}\left(\lambda=10^{-11.9} \mathrm{~s}^{-1}\right)$, respectively. The blue, green, claret, red, pink, and orange dashed lines represent the upper bounds given by LIGO, cantilever, LISA, cold atoms [31], bulk heating [33], and x-ray emission [39,40], respectively. Dark bars: The theoretical values suggested by Adler. Black dot and gray region: The GRW value and the theoretical lower bound [26,27].

\section{APPENDIX A: CALCULATION OF FLUCTUATION DYNAMICS}

The system was modeled by a classical mechanical oscillator with the motion described by the Langevin equations, which, in vectorial form, read

$$
m \ddot{\mathbf{x}}+m \Gamma \dot{\mathbf{x}}+\mathbf{K} \mathbf{x}+o\left(\mathbf{x}^{3}\right)=\mathbf{f}_{\mathrm{th}}(t)+\mathbf{f}_{\mathrm{CSL}}(t)+\mathbf{f}_{\mathrm{add}}(t),
$$

where $\mathbf{x}=(x, y, z), m$ is the mass of the oscillator, and $\boldsymbol{\Gamma}$ is the damping rate diagonal matrix with elements $\gamma_{i i}$ ( $i$ corresponding to $x, y$, and $z$ ). When the background gas damping dominates, $\gamma_{i i}$ are isotropic: $\gamma_{i i}=\gamma$. Similarly, $\mathbf{K}$ is the diagonal matrix of the effective spring constants with element $k_{i}=m \omega_{i}^{2}$ and $\omega_{i}$ is the resonance frequency of the oscillator along the $i$ th axis. $o\left(\mathbf{x}^{3}\right)$ includes the higher-order terms beyond the linear oscillator, such as Duffing nonlinearity $\alpha_{i} x_{i}^{3}$ and nonlinear couplings between different motions as $\beta_{i, j} x_{i} x_{j}^{2}$, etc. [83]. The right-hand side of the equation is a sum of force noises. They include the thermal fluctuations $f_{\text {th }}(t)$, possibly the CSL induced stochastic force $f_{\mathrm{CSL}}(t)$, and all the additional contributions, e.g., those due to the optical measurements, mechanical vibrations, etc.

Considering the motion in a single direction and dropping the direction label $i$, we estimate the three contributions to the noise in the system. The first one, the thermal force noise, was estimated by using the fluctuation dissipation theorem which gives the relation $\left\langle f_{\text {th }}(t) f_{\text {th }}(0)\right\rangle=2 m \gamma k_{\mathrm{B}} T_{\text {env }} \delta(t)$, where $T_{\text {env }}$ is environmental temperature. Equivalently, its strength can be described by the power spectral density $S_{\mathrm{th}}(\omega)=2 m \gamma k_{\mathrm{B}} T_{\mathrm{env}}$. The second contribution, $f_{\mathrm{CSL}}$, has been described in the main text. Within the third contribution, $f_{\text {add }}$, the optical force noise is the dominant one. It can be written as $f_{\text {opt }}(t)=f_{\text {int }}(t)+$ $f_{\mathrm{sc}}(t)$. The first term $f_{\text {int }}(t)$ is the classical optical force due to intensity fluctuations, including both those from the intensity fluctuation $\delta I(t)$ and the position fluctuation of the light position $\mathbf{x}_{\text {opt }}(t)$ relative to the center of the magnetogravitational trap. The illumination light intensity fluctuation induced force can be expressed as $-\alpha^{\prime} \nabla \xi\left[\mathbf{x}_{0}\right] \delta I(t) / 4$ and the light spot position fluctuation induced force as $-\alpha^{\prime} I \nabla\left(\nabla \xi\left(\mathbf{x}_{0}\right) \cdot \delta \mathbf{x}_{\text {opt }}(t)\right)$, with $\mathbf{x}_{0}$ the trapping position, $\xi\left(\mathbf{x}_{0}\right)$ the normalized light field distribution function, and $I$ the average intensity of the illumination light. The second term $f_{\mathrm{sc}}(t)$ is the stochastic force due to photon scattering. An additional contribution to $f_{\text {add }}$ is the parametric noise that is generated from the illumination light intensity, which leads to a fluctuation of the spring constant $k$ via optical force, and is proportional to $\delta I(t)$ [84].

We solved the Fokker-Planck equation for the probability density to obtain the statistical behavior of the system. To this end, the Langevin equations of motion in a single direction are written as

$$
m \ddot{x}+m \gamma \dot{x}+m \omega_{0}^{2} x+m \omega_{0}^{2} \zeta(t) x=f_{\text {total }}(t),
$$

where the parametric fluctuation $\zeta(t)$ was approximately taken as a white noise satisfying $\langle\zeta(t) \zeta(0)\rangle=\varsigma \delta(t)$. $f_{\text {total }}(t)=f_{\text {th }}(t)+f_{\mathrm{CSL}}(t)+f_{\text {opt }}(t)$ is the total force noise and it was also assumed to be white, $\left\langle f_{\text {total }}(t) f_{\text {total }}(0)\right\rangle=$ $2 m \gamma k_{\mathrm{B}} T_{\text {eff }} \delta(t)$, with $T_{\text {eff }}$ the effective temperature. It is noted that $f_{\text {total }}(t)$ and $\zeta(t)$ are not strictly independent, because both contain the contribution from the illumination light intensity 
fluctuation $\delta I(t)$. However, such a contribution in $f_{\text {total }}(t)$ is small, and so we took the total force noise and the parametric noise as approximately independent. Given this, we write the Langevin equations of motion as follows [85],

$$
\begin{aligned}
& d x=\frac{p}{m} d t, \\
& d p=\left(-m \omega_{0}^{2} x-\gamma p\right) d t+\sqrt{2 m \gamma k_{\mathrm{B}} T_{\mathrm{eff}}} d X+m \omega_{0}^{2} \varsigma x d Y,
\end{aligned}
$$

with $d X$ and $d Y$ two independent random variables with a Gaussian distribution. Setting the energy of the oscillator as $\varepsilon=p^{2} / 2 m+k x^{2} / 2$, with a high quality factor $Q=\omega_{0} / \gamma$, the Langevin equations of motion leads to the Fokker-Planck equation for the probability density $P(\varepsilon, t)$ which reads

$$
\begin{aligned}
\frac{\partial P(\varepsilon, t)}{\partial t}= & -\frac{\partial}{\partial \varepsilon}\left[\left(-\gamma \varepsilon+\gamma k_{\mathrm{B}} T_{\mathrm{eff}}\right) P(\varepsilon, t)\right] \\
& +\frac{\partial^{2}}{\partial \varepsilon^{2}}\left[\left(\gamma k_{\mathrm{B}} T_{\mathrm{eff}} \varepsilon+\frac{1}{4} \omega_{0}^{2} \varsigma^{2} \varepsilon^{2}\right) P(\varepsilon, t)\right] .
\end{aligned}
$$

For a stationary probability distribution $\partial P(\varepsilon, t) / \partial t=0$, and Eq. (A4) is

$$
P\left(x^{2}\right)=\frac{\gamma+\frac{1}{2} \omega_{0}^{2} \varsigma^{2}}{\gamma k_{\mathrm{B}} T_{\mathrm{eff}}}\left(1+\frac{m \omega_{0}^{4} \varsigma^{2}}{2 \gamma k_{\mathrm{B}} T_{\mathrm{eff}}} x^{2}\right)^{-\frac{2\left(\gamma+\omega_{0}^{2} \varsigma^{2}\right)}{\omega_{0}^{2} \varsigma^{2}}} .
$$

This distribution was measured experimentally. It is noted that, for the limiting case $\varsigma \rightarrow 0$, the expression in Eq. (A5) approaches the Gaussian distribution.

\section{APPENDIX B: DESIGN OF THE MAGNETOGRAVITATIONAL TRAP}

The potential energy density of a small diamagnetic microsphere in a magnetogravitational trap under an illumination light field can be written as [86]

$$
U(\mathbf{x})=-\frac{\chi}{2 \mu_{0}} V|\boldsymbol{B}(\mathbf{x})|^{2}+m g z+\frac{\alpha^{\prime}}{4} I V \xi(\mathbf{x})+U_{0} .
$$

Here, the first term is the diamagnetic potential, with $\chi$ and $v$ the magnetic susceptibility and volume of the microsphere; the second term is the gravitational potential, with $m$ the mass of the microsphere and $z$ is taken opposite to the direction of gravity; the third term is the optical gradient force, with $\alpha^{\prime}$ the real component of the polarizability, $I$ the light field intensity proportional to the light power, and $\xi(x)$ the normalized light field distribution function. The conditions that a diamagnetic microsphere can be stably trapped in the equilibrium position $\mathbf{x}_{\mathbf{0}}$ are

$$
\begin{gathered}
\mathbf{F}\left(x_{\mathbf{0}}\right)=-\nabla U\left(\mathbf{x}_{\mathbf{0}}\right)=\mathbf{0}, \\
\nabla \cdot \mathbf{F}\left(\mathbf{x}_{\mathbf{0}}\right)<\mathbf{0},
\end{gathered}
$$

with $\mathbf{F}(\mathbf{x})$ the total force of the potential. Near the equilibrium position $\mathbf{x}_{\mathbf{0}}$, the potential can be approximately expressed in quadratic form with respect to the displacement $\mathbf{x}$ from $\mathbf{x}_{\mathbf{0}}$ as $U\left(\mathbf{x}+\mathbf{x}_{\mathbf{0}}\right) \approx \Sigma_{i, j} \frac{\partial^{2} U\left(\mathbf{x}_{0}\right)}{\partial x_{i} \partial x_{j}} x_{i} x_{j}(i, j=x, y, z)$, which can be put into a diagonalized form as the sum of three independent

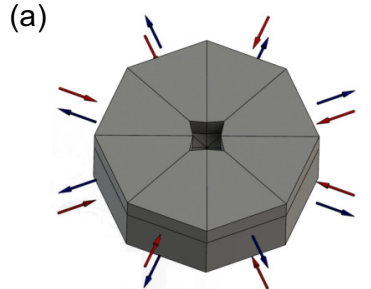

(b)
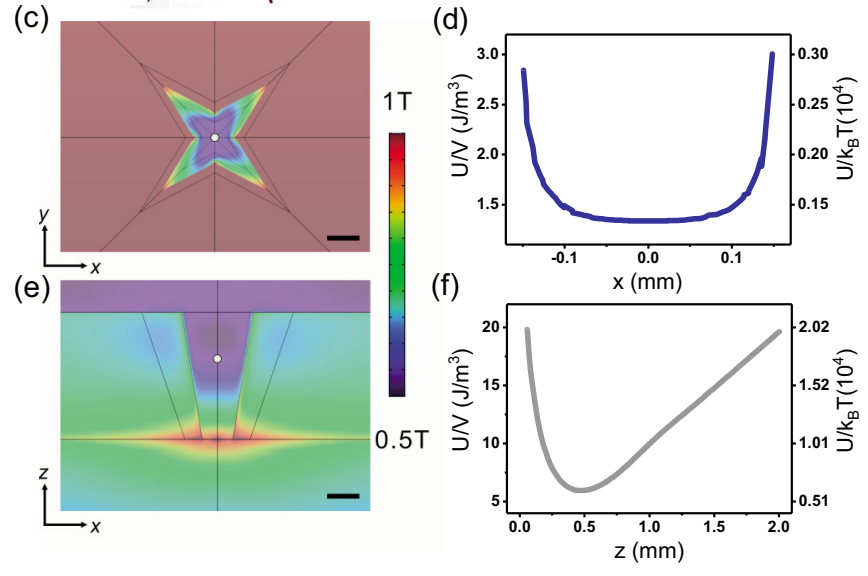

FIG. 5. Design of the magnetogravitational trap. (a) Schematic diagram of the trap. It consists of two layers of magnets with opposite magnetization; the arrows point in the direction of the north magnetic pole. (b) Image of the central portion of the trap used in this experiment with an area of about $1 \mathrm{~mm} \times 1 \mathrm{~mm}$. (c) and (e) Geometry and simulated magnetic field strength $|\boldsymbol{B}|$ of the trap in $x-y$ and $z-x$ planes (the scale bar equals $200 \mu \mathrm{m}$ ). The gray point shows the position where the microsphere is trapped. The microsphere is made of polyethylene glycol material with density $\mu=1.1 \times 10^{3} \mathrm{~kg} / \mathrm{m}^{3}$ and a magnetic susceptibility $\chi=-9.1 \times 10^{-6}$. (d) and (f) Calculated magnetogravitational potential energy density (left axes) as a function of the $x$ and $z$ coordinates, respectively. On the right axes, we report the corresponding energy (divided by $k_{\mathrm{B}} T_{\text {env }}$ ) for a microsphere of diameter $2 \mu \mathrm{m}$.

harmonic oscillators,

$$
U\left(\mathbf{x}+\mathbf{x}_{\mathbf{0}}\right) \approx \sum_{i} \frac{1}{2} k_{i} x_{i}^{2},
$$

where $k_{i}$ with $i=x, y, z$ are the effective spring constants, leading to the characteristic frequencies of the oscillators $\omega_{i}=$ $\sqrt{k_{i} / m}$. The constant term $U_{0}$ is dropped for convenience. The optical field will also generate an effective potential via the optical force, however, such an effect is much smaller than $U(\mathbf{x})$ and can be neglected. Hence, in the trap design, only the magnetic and the gravitational energies were taken into account. The potential function was calculated using a finite element simulation and the result is plotted in Fig. 5.

\section{APPENDIX C: EXPERIMENTAL SETUP AND MICROSPHERE GENERATION}

The experimental apparatus is shown in Fig. 6(a): The magnetogravitational trap is held in a vacuum system by specially designed springs, with the temperature of the inner chamber monitored and controlled to be slightly above room temperature, and the pressure controlled by a turbomolecular 

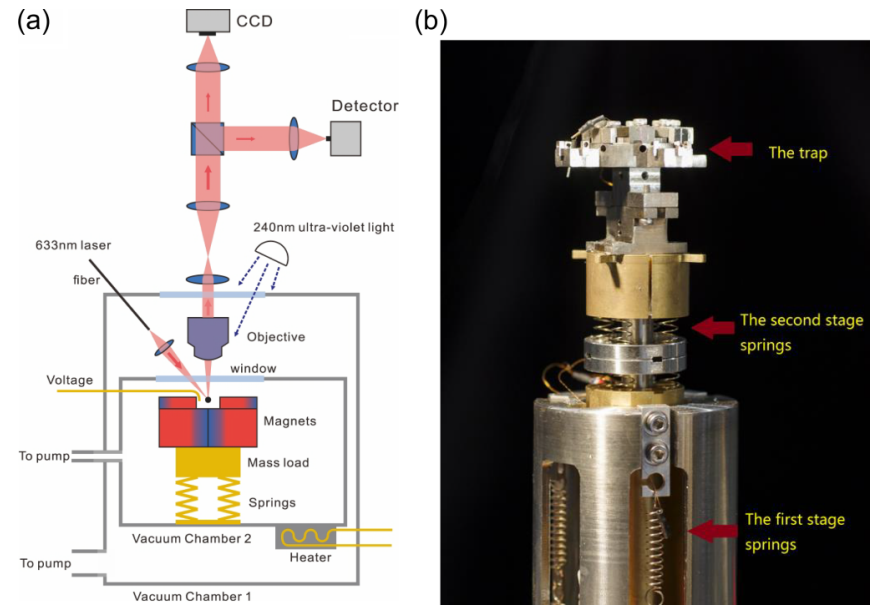

FIG. 6. Experimental setup. (a) A double layered vacuum chamber is used for temperature control. The environmental temperature of the inner chamber is maintained constant by PID feedback, and is kept slightly higher than room temperature by using a heater in the outer chamber. Ultraviolet light is used to eliminate the charge on the microsphere and an electrode in the trap in used to test the charge by applying a voltage. A 633-nm laser is applied to the microsphere, and the scattered light is collected by a CCD camera; the position and motion of the microsphere is recorded by a photon detector. The set of magnets forming the trap is mounted on a heavy copper frame, which is suspended via springs to isolate external vibrations. (b) Image of the copper frame with the vibration isolation system consisting of a two-stage spring-mass based suspension.

pump of tunable rotation speed. A CCD camera was used to detect the position of the microsphere, the magnification $\mathcal{M}$ of the detection optics being calibrated by a standard microstructure so that the displacement in the $x-y$ plane of the microsphere is $\mathbf{x}=\mathbf{x}^{\prime} / \mathcal{M}$, where $\mathbf{x}^{\prime}$ is the displacement of the microsphere image read out by the CCD camera. In this way, the thermal distribution was obtained.

A photodetector was used to detect the position-dependent scattering light intensity $I_{\mathrm{sc}}$, which is proportional to the illumination light $I$ as $I_{\mathrm{sc}} \propto I \xi\left(\mathbf{x}+\mathbf{x}_{\mathbf{0}}\right)$. Since the thermal motion is much bigger than the wavelength, such a detection scheme is efficient. The power spectral density in the position $S_{x}(\omega)$ is then calculated from the output photon detector voltage, $S_{x}(\omega) \propto S_{V}(\omega)$, with $S_{V}(\omega)$ the power spectral density of the output voltage. For high quality factor oscillators, the detection nonlinearity does not influence the results.

In order to eliminate the influence of the external vibration, the whole experimental setup is first mounted on an optical table with air legs, and a two-stage spring-mass based suspension is used to further isolate the vibrations, as shown in Fig. 6(b). The resonance frequency of the first stage (the second stage) in the $x-y$ plane is about $1.5 \mathrm{~Hz}(4 \mathrm{~Hz})$, and the mass of the first stage is designed to be much heavier than the mass of the second one. We used a very thin wire with a diameter about $40 \mu \mathrm{m}$ to apply the electric field which was used to pull the microsphere, and the wire was mechanically bounded on the first and then second stage before going to the trap, so that vibrations transmitted through the wire to the trap were effectively suppressed.
The microsphere used in our experiment is a small polyethylene glycol 400 droplet. To generate such a droplet with a desirable diameter, we first mixed polyethylene glycol 400 with dibutyl sebacate (DBS) and ethanol in a proportion of 1:27:1000 (volume ratio). Subsequently, droplets of the suspension were sprayed into the trap using a homebuilt piezoatomizer at atmospheric pressure. Ethanol rapidly evaporated after some seconds and a droplet with a typical diameter of 3-7 $\mu \mathrm{m}$ was obtained. Next, a moderated voltage of about a few tens of $\mathrm{V}$ was applied while the displacement of the droplet was monitored, and an ionizing radiation source (americium-241) was brought near the droplet. After exposing the droplet to the radiation for a few seconds, the charge on it changed randomly. Once a positively charged droplet was obtained, the pressure was gradually decreased to $10^{-6} \mathrm{mbar}$ for 1 day, and then DBS fully evaporated and the diameter of the microsphere no longer changed. Next, an ultraviolet light was used to slowly eliminate the positive charge until the droplet became fully neutralized. This was determined as follows: For a microsphere with only a few electron charges, jumps in the voltage-displacement response became clear, and eventually the responses dropped to zero when the net charge went to zero by applying a voltage larger than $50 \mathrm{~V}$. We also observed that the charge state was stable in vacuum $\left(P<10^{-4}\right.$ mbar) for a very long time (tens of days or even longer).

\section{APPENDIX D: INFLUENCE OF NONLINEARITY ON MEASUREMENT RESULTS}

The nonlinear term in Eq. (A1) becomes important for a motion with large amplitude. For simplicity, we consider the term $\alpha x^{3}$ but temporarily we omit the coupling terms $\beta_{i, j} x_{i}^{2} x_{j}$; the oscillator then becomes a Duffing oscillator with the following equation of motion [83],

$$
m \ddot{x}+m \gamma \dot{x}+k x+\alpha x^{3}=f_{\text {total }}(t),
$$

where $\alpha$ is the Duffing constant. One of the important effects of nonlinearity is a frequency shift and broadening that are proportional to the thermal fluctuation $\alpha k_{\mathrm{B}} T_{\mathrm{eff}}$ [87]. When such a nonlinear thermal broadening becomes larger than the damping rate $\gamma$, the power spectral density shows a non-Lorentz character [75]. Hence, in the thermal nonlinear regime, the damping rate $\gamma / 2 \pi$ cannot be obtained by measuring the full width at half maximum based on the power spectral density, which is commonly used with a harmonic oscillator. Instead, we notice that the change of energy over time is still the same as that of a harmonic oscillator. This is because the reduction of energy in the damping process results from the dissipation via the kinetic energy $p^{2} / 2 m$, while the nonlinearity only modifies the potential energy and preserves energy conservation [76]. Therefore, we extract $\gamma$ from the energy autocorrelation as described below.

From Eq. (D1), we first write equations of motion for position and momentum, without the fluctuation $f_{\text {total }}(t)$, i.e.,

$$
\begin{aligned}
d x & =\frac{p}{m} d t, \\
d p & =\left(-k x-\alpha x^{3}-\gamma p\right) d t .
\end{aligned}
$$


Then the change of the total energy of the oscillator follows

$$
d \varepsilon=\frac{\partial \varepsilon}{\partial x} d x+\frac{\partial \varepsilon}{\partial p} d p=-\frac{\gamma p^{2}}{m} d t .
$$

Next, we consider a short period during which the dissipation is negligible so the motion of the system can be written as

$$
x(t)=X(t)\left[\cos (\omega t)+\frac{\kappa X^{2}(t)}{12} \cos (3 \omega t)+o\left(\kappa^{2} X^{4}(t)\right)\right] .
$$

Here, $X(t)$ is a vibrational amplitude that is slowly varying, $\kappa=3 \alpha / 8 m \omega_{0}^{2}$, and $\omega$ is an amplitude-dependent oscillation frequency which shifts from the resonance frequency $\omega_{0}$ as

$$
\omega=\omega_{0}\left(1+\kappa X^{2}(t)\right) .
$$

As $X(t)$ goes to zero, we have $x(t) \approx X(t) \cos (\omega \mathrm{t})$, as expected.

Then, we define the average kinetic energy $E_{K}$ and average potential energy $V$ as

$$
\begin{gathered}
E_{K}=\frac{1}{\tau} \int_{0}^{\tau} \frac{1}{2} m \dot{x}^{2}(t) d t, \\
V=\frac{1}{\tau} \int_{0}^{\tau}\left[\frac{1}{2} m \omega_{0}^{2} x^{2}(t)+\frac{1}{4} \alpha x^{4}(t)\right] d t,
\end{gathered}
$$

with $\tau$ much shorter than $1 / \gamma$ but much longer than $1 / \omega$, which can be satisfied for a system with a large quality factor $Q=\omega / \gamma$. By averaging Eq. (D3) as $d \varepsilon / d t=-2 \gamma E_{K}$, we obtain the differential equation for $X^{2}(t)$,

$$
\frac{d X^{2}(t)}{d t}=-\gamma X^{2}(t)\left[1-\kappa X^{2}(t)+O\left(\kappa^{2} X^{4}(t)\right)\right] .
$$

Dropping terms of order $\kappa^{2} X^{4}(t)$ or higher, we obtain the solution of Eq. (D8),

$$
X^{2}(t)\left[\kappa-\left(\frac{\kappa X^{2}(0)-1}{X^{2}(0)}\right) e^{\gamma t}\right]=1,
$$

Asymptotically, $X(t)$ decays and Eq. (D9) can be expanded as follows,

$$
X^{2}(t)=X^{2}(0) e^{-\gamma t}\left[1-\kappa X^{2}(0)\left(e^{-\gamma t}-1\right)+\cdots\right] .
$$

Next, we define the autocorrelation function of $X^{2}(t)$ as:

$$
G_{X^{2}}(t)=\left\langle X^{2}(t) X^{2}(0)\right\rangle,
$$

which according to Eq. (D10) becomes

$$
G_{X^{2}}(t)=\left\langle X^{4}(0)\right\rangle e^{-\gamma t}\left[1-\kappa X^{2}(0)\left(e^{-\gamma t}-1\right)+\cdots\right] .
$$

In the experiment, $X^{2}(t)$ is directly measured from the power spectral density $S_{x}(\omega)$ by following standard procedures [88,89], as $X^{2}(t)=S_{x}(\omega) b$, where $b$ is the sampling bandwidth satisfying $\gamma \ll b \ll \omega_{0}$. We also define the following normalized autocorrelation,

$$
R_{X^{2}}(t)=\frac{\left\langle X^{2}(t) X^{2}(0)\right\rangle}{\left\langle X^{4}(0)\right\rangle},
$$

which is used to estimate the damping rate $\gamma / 2 \pi$.

In our system, nonlinearities come not only from the term $\alpha x^{3}$, but also from the coupling of the motion along different
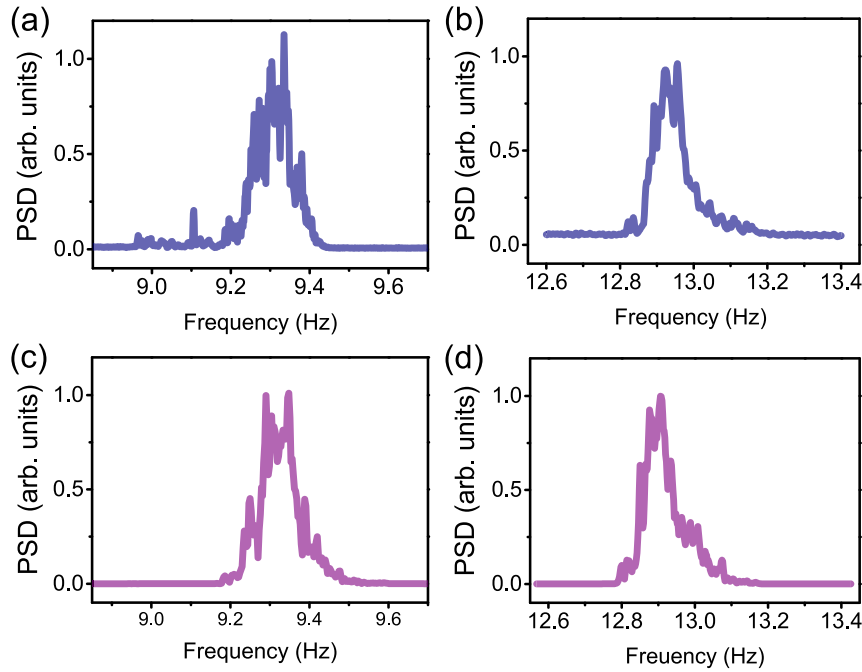

FIG. 7. Power spectral density (PSD) of the displacement $S_{\mathrm{x}}(\omega)$ under high vacuum. (a) and (b) Measured data of two different oscillation modes corresponding to the resonance frequencies $\omega_{2} / 2 \pi \approx$ $9.3 \mathrm{~Hz}$ and $\omega_{1} / 2 \pi \approx 12.9 \mathrm{~Hz}$; the full width at half maximum of the peak turns out to be much larger than $\gamma / 2 \pi$ and asymmetric, which can be explained by the nonlinearity of the trap. (c) and (d) Numerical simulations of (a) and (b) by introducing a nonlinearity, where the nonlinear coefficients are adjusted so that simulation and experiment agree with each other.

axes, as $\beta_{i, j} x_{i} x_{j}^{2}$. We calculated numerically the effects based on two-mode coupling from the equations of motion,

$$
\begin{aligned}
& m \ddot{x}_{1}+m \gamma \dot{x}_{1}+m \omega_{1}^{2} x_{1}+\alpha_{1} x_{1}^{3}+\beta x_{2}^{2} x_{1}=f_{1}(t), \\
& m \ddot{x}_{2}+m \gamma \dot{x}_{2}+m \omega_{2}^{2} x_{2}+\alpha_{2} x_{2}^{3}+\beta x_{1}^{2} x_{2}=f_{2}(t) .
\end{aligned}
$$

Here, modes 1 and 2 correspond to the motions in the $x-y$ plane, while the motion along the $z$ axis is neglected, and $f_{1}(t)$ and $f_{2}(t)$ are independent white noise with a power spectral density $S_{1,2}$ equal to that of the thermal Brownian noise measured experimentally. The values $m, \gamma$, and $\omega_{1,2}$ are directly obtained from the experiment. The nonlinearity coefficients $\alpha_{1,2}$ and $\beta$ are tuned so that the full width at half maximum and the shape of the power spectral density $S_{x}(\omega)$ obtained from the numerical simulation and from the experiments agree with each other, as shown in Fig. 7. The corresponding values are $\alpha_{1}=-6.4 \mathrm{~kg} / \mathrm{m}^{2} \mathrm{~s}^{2}, \alpha_{2}=-2.1 \mathrm{~kg} / \mathrm{m}^{2} \mathrm{~s}^{2}$, and $\beta=$ $6.4 \mathrm{~kg} / \mathrm{m}^{2} \mathrm{~s}^{2} . R_{X^{2}}(t)$ is numerically calculated for medium and high vacuum and the results are shown in Fig. 8. The data are fitted to the exponential decay $\exp (-t / \tau)$, producing the damping rates $\gamma=1 / \tau$, which agree well with the values used in numerical simulations (see Table II).

\section{APPENDIX E: ERROR ESTIMATION}

In order to estimate the error on the effective temperature $T_{\text {eff }}$ from the measured position distribution, the displacement distributions of the oscillation mode $1(12.9 \mathrm{~Hz})$ under high vacuum (HV) and medium vacuum (MV) were recorded [Figs. 9(a) and 9(b)]. The results are fitted to a Gaussian distribution to give the standard deviation $\sigma^{\mathrm{HV}}\left(\sigma^{\mathrm{MV}}\right)$, from which the effective temperatures $T_{\text {eff }}^{\mathrm{HV}}\left(T_{\text {eff }}^{\mathrm{MV}}\right)$ are obtained. For a given measurement time $t_{\text {mea }}$, the standard deviation 

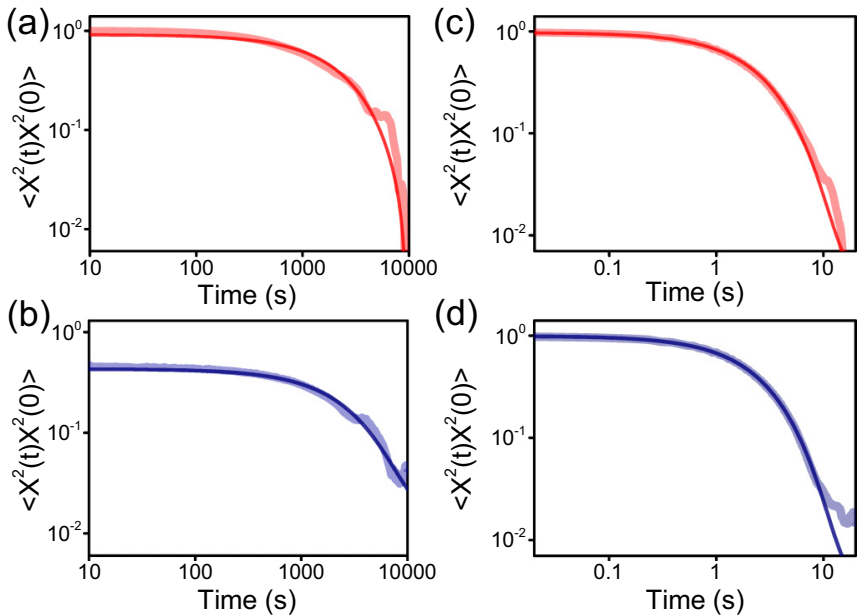

FIG. 8. Influence of the nonlinearity on the autocorrelation of the oscillator energy defined as $\left\langle X^{2}(t) X^{2}(0)\right\rangle$ [normalized as per Eq. (D13)]. (a) and (b) Numerically simulated autocorrelation (light thick curves) under high vacuum (with $\gamma / 2 \pi=0.0004 \mathrm{~Hz}$ ) when the nonlinearity is excluded and included, respectively. The curves are fitted with the exponential decay $\exp (-t / \tau)$ (thin curves); the resulting damping rates $\gamma$ turn out to be almost the same as the input ones (see Table II for the values). (c) and (d) Medium vacuum counterparts of (a) and (b), with a larger damping rate $\gamma / 2 \pi=$ $0.4 \mathrm{~Hz}$. The recovered damping rates with and without nonlinearity both agree well with the input values.

$\sigma_{T_{\text {eff }}}$ of the measured effective temperature can be derived by following the procedure in Refs. [90,91]. The results for medium vacuum (MV) and high vacuum (HV) are plotted in Figs. 9(c) and 9(d), respectively, as functions of $t_{\text {mea }}$. Theoretically, the relative standard deviation of the effective temperature as a function of the measurement time $t_{\text {mea }}$ satisfies the relation [91]

$$
\frac{\sigma_{T_{\text {eff }}\left(t_{\text {mea }}\right)}}{T_{\text {eff }}}=\sqrt{\frac{2}{\gamma t_{\text {mea }}}},
$$

and is plotted in Figs. 9(c) and 9(d) as straight lines. The measured data agree very well with the theory. Finally, the uncertainty $\sigma_{T_{\text {eff }}}$ of the effective temperature is estimated using Eq. (E1) by taking $t_{\text {mea }}=t_{\text {total }}$, the total measurement time. In particular, the total data acquisition time at high vacuum is $9.5 \times 10^{5} \mathrm{~s}$ (about 11 days), which can be further extended to reduce the uncertainty, but this was not done for practical

TABLE II. Comparison of the damping rates. Input $\gamma$ is the input value of damping rate used in the simulation, fitted $\gamma$ (nonlinear) is the result of the simulation with nonlinearity added into the equation of motion, and fitted $\gamma$ (linear) that from the result of simulation without nonlinearity. The first row corresponds to medium vacuum, while the second row to high vacuum.

\begin{tabular}{lccc}
\hline \hline Input $\gamma(\mathrm{Hz})$ & $\begin{array}{c}\text { Fitted } \gamma \\
\text { (nonlinear) }(\mathrm{Hz})\end{array}$ & $\begin{array}{c}\text { Fitted } \gamma \\
\text { (linear) }(\mathrm{Hz})\end{array}$ \\
\hline$P_{\mathrm{MV}}$ & 0.4 & 0.39 & 0.38 \\
$P_{\mathrm{HV}}$ & 0.0004 & 0.00037 & 0.00038 \\
\hline \hline
\end{tabular}
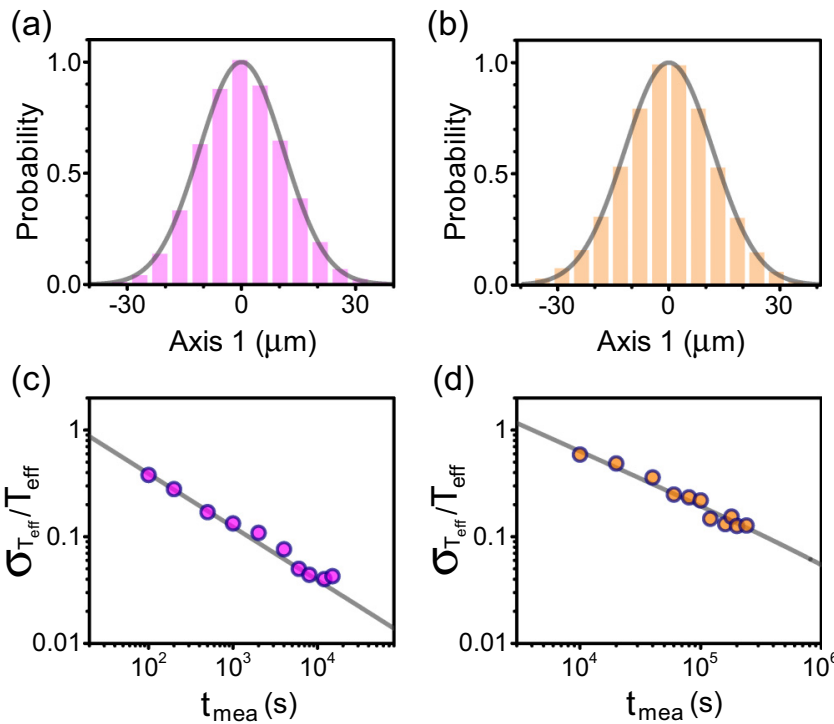

(d)

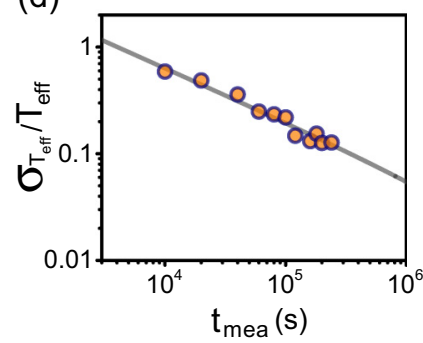

FIG. 9. (a) Probability distribution of the displacement, measured in medium vacuum $\left(P \approx 1 \times 10^{-4}\right.$ mbar $)$, and its fitting with a Gaussian distribution function of standard deviation $\sigma$. (b) Same as (a) but measured in high vacuum $\left(P \approx 5 \times 10^{-7}\right.$ mbar $)$. (c) Standard deviation of the relative effective temperature $\sigma_{T_{\text {eff }}}\left(t_{\text {mea }}\right) / T_{\text {eff }}$ as a function of acquisition time $t_{\text {mea }}$ at medium vacuum. The total measurement time is about $7.5 \times 10^{4} \mathrm{~s}$. The curve shows the theoretical value according to Eq. (E1). (d) Same as (c) but measured at high vacuum. The total measurement time is about $9.5 \times 10^{5} \mathrm{~s}$.

reasons. The effective temperature measured in a medium vacuum is taken as the environmental temperature $T_{\mathrm{eff}}^{\mathrm{MV}}=T_{\mathrm{env}}$ and the temperature difference is $\delta T=T_{\text {eff }}^{\mathrm{HV}}-T_{\text {eff }}^{\mathrm{MV}}$. To estimate the upper bound on $\delta T$ with the standard methods [92], $T_{\text {eff }}^{\mathrm{HV}}$ and $T_{\text {eff }}^{\mathrm{MV}}$ are treated as independent and both following Gaussian distributions, with their corresponding standard deviations $\sigma_{T_{\text {eff }}}$ obtained from the measured data [Figs. $9(\mathrm{c})$ and 9(d)]. The threshold $\sigma_{\delta T}$ defined by the $95 \%$ confidence level $\left(\delta T<\sigma_{\delta T}\right)$ is given in Table III. We note that the measured effective temperature does not coincide with the temperature ( $298 \mathrm{~K})$ measured by the thermometer in the vacuum chamber. While such a bias is due to the uncertainty in measuring the absolute displacement of the oscillator, there is an uncertainty of less than a few percent in determining the magnification $\mathcal{M}$ of the detection optics, so is in the microsphere's absolute displacement is given by $\mathbf{x}=\mathbf{x}^{\prime} / \mathcal{M}$. This uncertainty is constant during the whole experimental process and only

TABLE III. Measured effective temperature of the oscillator $T_{\mathrm{eff}}^{\mathrm{HV}}$ is the effective temperature of the oscillator measured at high vacuum and $T_{\text {eff }}^{\mathrm{MV}}$ that at medium vacuum. $T_{\text {eff }}^{\mathrm{MV}}=T_{\text {env }}$ is used for the estimation of the environment temperature. $\delta T$ is defined as the temperature increase $\delta T=T_{\text {eff }}^{\mathrm{HV}}-T_{\text {env }} . \sigma_{T_{\mathrm{eff}}^{\mathrm{HV}}}, \sigma_{T_{\mathrm{eff}}^{\mathrm{MV}}}$, and $\sigma_{\delta T}$ are the corresponding upper bounds at a $95 \%$ confidence level.

\begin{tabular}{|c|c|c|c|c|c|}
\hline \multicolumn{2}{|c|}{ High vacuum } & \multicolumn{2}{|c|}{ Medium vacuum } & \multicolumn{2}{|c|}{ Difference } \\
\hline$T_{\mathrm{eff}}^{\mathrm{HV}}$ & $\sigma_{T_{\mathrm{eff}}^{\mathrm{HV}}}$ & $T_{\text {eff }}^{\mathrm{MV}}$ & $\sigma_{T_{\mathrm{eff}}^{\mathrm{MV}}}$ & $\delta T$ & $\sigma_{\delta T}$ \\
\hline $297.9 \mathrm{~K}$ & $16.2 \mathrm{~K}$ & $291.4 \mathrm{~K}$ & $4.1 \mathrm{~K}$ & $6.5 \mathrm{~K}$ & $40 \mathrm{~K}$ \\
\hline
\end{tabular}


brings about a small error (a few percent) on the final result. Since the power spectral density of additional force noise is defined as $\delta S_{\text {total }}=2 m \gamma k_{\mathrm{B}} \delta T$, we estimate its upper bound as $\delta S_{\text {total }}<2 m \gamma k_{\mathrm{B}} \sigma_{\delta T}$. Finally, we obtain the upper bounds on the CSL collapse rate $\lambda$ from Eq. (3) by using the upper bound on the CSL collapse strength $\eta$ given by $\hbar^{2} \eta<2 m \gamma k_{\mathrm{B}} \sigma_{\delta T}$.
[1] D. Bohm, A suggested interpretation of the quantum theory in terms of "Hidden" variables. I, Phys. Rev. 85, 166 (1952).

[2] D. Bohm, A suggested interpretation of the quantum theory in terms of "Hidden" variables. II, Phys. Rev. 85, 180 (1952).

[3] D. Dürr and S. Teufel, Bohmian Mechanics (Springer, Berlin, 2009).

[4] H. Everett, Theory of the universal wavefunction, Ph.D. thesis, Princeton University, 1956.

[5] H. Everett, "Relative state" formulation of quantum mechanics, Rev. Mod. Phys. 29, 454 (1957).

[6] D. Wallace, Everettian rationality: Defending Deutsch's approach to probability in the Everett interpretation, Stud. Hist. Philos. Mod. Phys. 34, 415 (2003).

[7] G. Chiribella, G. M. D'Ariano, and P. Perinotti, Informational derivation of quantum theory, Phys. Rev. A 84, 012311 (2011).

[8] G. C. Ghirardi, A. Rimini, and T. Weber, Unified dynamics for microscopic and macroscopic systems, Phys. Rev. D 34, 470 (1986).

[9] P. Pearle, Combining stochastic dynamical state-vector reduction with spontaneous localization, Phys. Rev. A 39, 2277 (1989).

[10] G. C. Ghirardi, P. Pearle, and A. Rimini, Markov processes in Hilbert space and continuous spontaneous localization of systems of identical particles, Phys. Rev. A 42, 78 (1990).

[11] A. Bassi and G. Ghirardi, Dynamical reduction models, Phys. Rep. 379, 257 (2003).

[12] A. Bassi, K. Lochan, S. Satin, T. P. Singh, and H. Ulbricht, Models of wave-function collapse, underlying theories, and experimental tests, Rev. Mod. Phys. 85, 471 (2013).

[13] S. Nimmrichter, K. Hornberger, and K. Hammerer, Optomechanical Sensing of Spontaneous Wave-Function Collapse, Phys. Rev. Lett. 113, 020405 (2014).

[14] M. Bahrami, M. Paternostro, A. Bassi, and H. Ulbricht, Proposal for a Noninterferometric Test of Collapse Models in Optomechanical Systems, Phys. Rev. Lett. 112, 210404 (2014).

[15] L. Diósi, Testing Spontaneous Wave-Function Collapse Models on Classical Mechanical Oscillators, Phys. Rev. Lett. 114, 050403 (2015).

[16] A. J. Leggett, Testing the limits of quantum mechanics: Motivation, state of play, prospects, J. Phys.: Condens. Matter 14, R415 (2002).

[17] L. Diósi, Models for universal reduction of macroscopic quantum fluctuations, Phys. Rev. A 40, 1165 (1989).

[18] R. Penrose, On gravity's role in quantum state reduction, Gen. Relat. Gravit. 28, 581 (1996).

[19] R. Penrose, The Road to Reality: A Complete Guide to the Laws of the Universe (Jonathan Cape, London, 2004).

[20] S. L. Adler, Lower and upper bounds on CSL parameters from latent image formation and IGM heating, J. Phys. A: Math. Theor. 40, 2935 (2007).

[21] T. Kovachy, P. Asenbaum, C. Overstreet, C. A. Donnelly, S. M. Dickerson, A. Sugarbaker, J. M. Hogan, and M. A.
Kasevich, Quantum superposition at the half-metre scale, Nature (London) 528, 530 (2015).

[22] M. Arndt, O. Nairz, J. Vos-Andreae, C. Keller, G. van der Zouw, and A. Zeilinger, Wave-particle duality of $\mathrm{C}_{60}$ molecules, Nature (London) 28, 680 (1999).

[23] S. Gerlich, S. Eibenberger, M. Tomandl, S. Nimmrichter, K. Hornberger, P. J. Fagan, J. Tüxen, M. Mayor, and M. Arndt, Quantum interference of large organic molecules, Nat. Commun. 2, 263 (2011)

[24] K. Hornberger, S. Gerlich, P. Haslinger, S. Nimmrichter, and M. Arndt, Quantum interference of clusters and molecules, Rev. Mod. Phys. 84, 157 (2012).

[25] S. Eibenberger, S. Gerlich, M. Arndt, M. Mayor, and J. Tüxen, Matter-wave interference of particles selected from a molecular library with masses exceeding $10000 \mathrm{amu}$, Phys. Chem. Chem. Phys. 15, 14696 (2013).

[26] M. Toroš, G. Gasbarri, and A. Bassi, Colored and dissipative continuous spontaneous localization model and bounds from matter-wave interferometry, Phys. Lett. A 381, 3921 (2017).

[27] M. Toroš and A. Bassi, Bounds on quantum collapse models from matter-wave interferometry: Calculational details, J. Phys. A: Math. Theor. 51, 115302 (2018).

[28] S. Belli, R. Bonsignori, G. D’Auria, L. Fant, M. Martini, S. Peirone, S. Donadi, and A. Bassi, Entangling macroscopic diamonds at room temperature: Bounds on the continuousspontaneous-localization parameters, Phys. Rev. A 94, 012108 (2016).

[29] K. C. Lee, M. R. Sprague, B. J. Sussman, J. Nunn, N. K. Langford, X.-M. Jin, T. Champion, P. Michelberger, K. F. Reim, D. England, D. Jaksch, and I. A. Walmsley, Entangling macroscopic diamonds at room temperature, Science 334, 1253 (2011).

[30] T. Kovachy, J. M. Hogan, A. Sugarbaker, S. M. Dickerson, C. A. Donnelly, C. Overstreet, and M. A. Kasevich, Matter Wave Lensing to Picokelvin Temperatures, Phys. Rev. Lett. 114, 143004 (2015).

[31] M. Bilardello, S. Donadi, A. Vinante, and A. Bassi, Bounds on collapse models from cold-atom experiments, Physica A 462, 764 (2016).

[32] E. Nazaretski, V. O. Kostroun, S. Dimov, R. O. Pohl, and J. M. Parpia, Heat inputs to sub-mK temperature cryostats and experiments from $\gamma$-radiation and cosmic ray muons, J. Low Temp. Phys. 137, 609 (2004).

[33] S. L. Adler and A. Vinante, Bulk heating effects as tests for collapse models, Phys. Rev. A 97, 052119 (2018).

[34] M. Bahrami, Testing collapse models by a thermometer, Phys. Rev. A 97, 052118 (2018).

[35] I. de Pater and J. J. Lissauer, Planetary Sciences (Cambridge University Press, Cambridge, UK, 2001).

[36] S. L. Adler, A. Bassi, M. Carlesso, and A. Vinante, Testing continuous spontaneous localization with Fermi liquids, Phys. Rev. D 99, 103001 (2019). 
[37] A. Tilloy and T. M. Stace, Neutron Star Heating Constraints on Wave-Function Collapse Models, Phys. Rev. Lett. 123, 080402 (2019).

[38] A. Bassi and S. Donadi, Spontaneous photon emission from a non-relativistic free charged particle in collapse models: A case study, Phys. Lett. A 378, 761 (2014).

[39] C. Curceanu, B. C. Hiesmayr, and K. Piscicchia, X-rays help to unfuzzy the concept of measurement, J. Adv. Phys. 4, 263 (2015).

[40] K. Piscicchia, A. Bassi, C. Curceanu et al., CSL collapse model mapped with the spontaneous radiation, Entropy 19, 319 (2017).

[41] A. Vinante et al. (AURIGA Collaboration), Present performance and future upgrades of the AURIGA capacitive readout, Class. Quantum Grav. 23, S103 (2006).

[42] B. P. Abbott et al. (LIGO Scientific Collaboration and Virgo Collaboration), GW150914: The Advanced LIGO Detectors in the Era of First Discoveries, Phys. Rev. Lett. 116, 131103 (2016).

[43] M. Armano et al., Sub-Femto-g Free Fall for Space-Based Gravitational Wave Observatories: LISA Pathfinder Results, Phys. Rev. Lett. 116, 231101 (2016).

[44] M. Armano et al., Beyond the Required LISA Free-Fall Performance: New LISA Pathfinder Results down to $20 \mu \mathrm{Hz}$, Phys. Rev. Lett. 120, 061101 (2018)

[45] M. Carlesso, A. Bassi, P. Falferi, and A. Vinante, Experimental bounds on collapse models from gravitational wave detectors, Phys. Rev. D 94, 124036 (2016).

[46] B. Helou, B. J. J. Slagmolen, D. E. McClelland, and Y. Chen, LISA pathfinder appreciably constrains collapse models, Phys. Rev. D 95, 084054 (2017).

[47] A. Vinante, M. Bahrami, A. Bassi, O. Usenko, G. Wijts, and T. H. Oosterkamp, Upper Bounds on Spontaneous WaveFunction Collapse Models Using Millikelvin-Cooled Nanocantilevers, Phys. Rev. Lett. 116, 090402 (2016).

[48] A. Vinante, R. Mezzena, P. Falferi, M. Carlesso, and A. Bassi, Improved Noninterferometric Test of Collapse Models Using Ultracold Cantilevers, Phys. Rev. Lett. 119, 110401 (2017).

[49] A. Pontin, N. P. Bullier, M. Toroš, and P. F. Barker, An ultranarrow line width levitated nano-oscillator for testing dissipative wavefunction collapse, arXiv:1907.06046.

[50] B. Collett and P. Pearle, Wavefunction collapse and random walk, Found. Phys. 33, 1495 (2003).

[51] M. Carlesso, M. Paternostro, H. Ulbricht, A. Vinante, and A. Bassi, Non-interferometric test of the continuous spontaneous localization model based on the torsional motion of a cylinder, New J. Phys. 20, 083022 (2018).

[52] D. Goldwater, M. Paternostro, and P. F. Barker, Testing wavefunction-collapse models using parametric heating of a trapped nanosphere, Phys. Rev. A 94, 010104(R) (2016).

[53] R. Kaltenbaek, M. Aspelmeyer, and V. Vedral, Macroscopic Quantum Resonators (MAQRO): 2015 update, EPJ Quantum Technol. 3, 5 (2016).

[54] S. McMillen, M. Brunelli, M. Carlesso, A. Bassi, H. Ulbricht, M. G. A. Paris, and M. Paternostro, Quantum-limited estimation of continuous spontaneous localization, Phys. Rev. A 95, 012132 (2017).

[55] B. Schrinski, B. A. Stickler, and K. Hornberger, Collapseinduced orientational localization of rigid rotors, J. Opt. Soc. Am. B 34, C1 (2017).
[56] M. Carlesso, A. Vinante, and A. Bassi, Multilayer test masses to enhance the collapse noise, Phys. Rev. A 98, 022122 (2018).

[57] R. Mishra, A. Vinante, and T. P. Singh, Testing spontaneous collapse through bulk heating experiments: An estimate of the background noise, Phys. Rev. A 98, 052121 (2018).

[58] A. Bassi and L. Ferialdi, Non-Markovian dynamics for a free quantum particle subject to spontaneous collapse in space: General solution and main properties, Phys. Rev. A 80, 012116 (2009).

[59] L. Ferialdi and A. Bassi, Dissipative collapse models with nonwhite noises, Phys. Rev. A 86, 022108 (2012).

[60] S. L. Adler, A. Bassi, and S. Donadi, On spontaneous photon emission in collapse models, J. Phys. A 46, 245304 (2013).

[61] M. Carlesso, L. Ferialdi, and A. Bassi, Colored collapse models from the non-interferometric perspective, Eur. Phys. J. D 72, 159 (2018).

[62] M. Aspelmeyer, T. J. Kippenberg, and F. Marquardt, Cavity optomechanics, Rev. Mod. Phys. 86, 1391 (2014).

[63] A. Vinante, A. Pontin, M. Rashid, M. Toros, P. F. Barker, and H. Ulbricht, Testing collapse models with levitated nanoparticles: The detection challenge, Phys. Rev. A 100, 012119 (2019).

[64] O. Romero-Isart, Quantum superposition of massive objects and collapse models, Phys. Rev. A 84, 052121 (2011).

[65] Z.-q. Yin, T. Li, X. Zhang, and L. M. Duan, Large quantum superpositions of a levitated nanodiamond through spinoptomechanical coupling, Phys. Rev. A 88, 033614 (2013).

[66] J. Li, S. Zippilli, J. Zhang, and D. Vitali, Discriminating the effects of collapse models from environmental diffusion with levitated nanospheres, Phys. Rev. A 93, 050102(R) (2016).

[67] J. A. Sidles, J. L. Garbini, K. J. Bruland, D. Rugar, O. Züger, S. Hoen, and C. S. Yannoni, Magnetic resonance force microscopy, Rev. Mod. Phys. 67, 249 (1995).

[68] A. A. Clerk, M. H. Devoret, S. M. Girvin, F. Marquardt, and R. J. Schoelkopf, Introduction to quantum noise, measurement, and amplification, Rev. Mod. Phys. 82, 1155 (2010).

[69] P. Huang, P. Wang, J. Zhou, Z. Wang, C. Ju, Z. Wang, Y. Shen, C. Duan, and J. Du, Demonstration of Motion Transduction Based on Parametrically Coupled Mechanical Resonators, Phys. Rev. Lett. 110, 227202 (2013).

[70] D. Rugar, R. Budakian, H. J. Mamin, and B. W. Chui, Single spin detection by magnetic resonance force microscopy, Nature (London) 430, 329 (2004).

[71] E. Gavartin, P. Verlot, and T. J. Kippenberg, A hybrid on-chip optomechanical transducer for ultrasensitive force measurements, Nat. Nanotechnol. 7, 509 (2012).

[72] A. K. Geim, M. D. Simon, M. I. Boamfa, and L. O. Heflinger, Magnet levitation at your fingertips, Nature (London) 400, 323 (1999).

[73] B. R. Slezak, C. W. Lewandowski, J. Hsu, and B. D'Urso, Cooling the motion of a silica microsphere in a magnetogravitational trap in ultra-high vacuum, New J. Phys. 20, 063028 (2018).

[74] D. E. Chang, C. A. Regal, S. B. Papp, D. J. Wilson, J. Yeb, O. Painterd, H. J. Kimble, and P. Zoller, Cavity opto-mechanics using an optically levitated nanosphere, Proc. Natl. Acad. Sci. USA 107, 1005 (2010).

[75] M. I. Dykman and P. V. E. McClintock, Power spectra of noisedriven nonlinear systems and stochastic resonance, Physica D 58, 10 (1992). 
[76] A. Juyal, A. Agarwal, and S. Mukhopadhyay, Noncontact Friction and Force Fluctuations between Closely Spaced Bodies, Phys. Rev. Lett. 120, 096801 (2018).

[77] J. Gieseler, L. Novotny, and R. Quidant, Thermal nonlinearities in a nanomechanical oscillator, Nat. Phys. 9, 806 (2013).

[78] J. Millen, P. Z. G. Fonseca, T. Mavrogordatos, T. S. Monteiro, and P. F. Barker, Cavity Cooling a Single Charged Levitated Nanosphere, Phys. Rev. Lett. 114, 123602 (2015).

[79] T. Li, S. Kheifets, and M. G. Raizen, Millikelvin cooling of an optically trapped microsphere in vacuum, Nat. Phys. 7, 527 (2011).

[80] H. Pino, J. Prat-Camps, K. Sinha, B. Prasanna Venkatesh, and O. Romero-Isart, On-chip quantum interference of a superconducting microsphere, Quantum Sci. Technol. 3, 25001 (2018).

[81] O. Romero-Isart, L. Clemente, C. Navau, A. Sanchez, and J. I. Cirac, Quantum Magnetomechanics with Levitating Superconducting Microspheres, Phys. Rev. Lett. 109, 147205 (2012).

[82] M. Cirio, G. K. Brennen, and J. Twamley, Quantum Magnetomechanics: Ultrahigh- $Q$-Levitated Mechanical Oscillators, Phys. Rev. Lett. 109, 147206 (2012).

[83] R. Lifshitz and M. C. Cross, Review of Nonlinear Dynamics and Complexity (Wiley-VCH, Weinheim, 2009).

[84] J. Gieseler, B. Deutsch, R. Quidant, and L. Novotny, Subkelvin Parametric Feedback Cooling of a Laser-Trapped Nanoparticle, Phys. Rev. Lett. 109, 103603 (2012).
[85] N. G. van Kampen, Stochastic differential equations, Phys. Rep. 24, 171 (1976).

[86] M. V. Berry and A. K. Geim, Of flying frogs and levitrons, Eur. J. Phys. 18, 307 (1997).

[87] M. Dykman and M. Krivoglaz, Fluctuations in nonlinear systems near bifurcations corresponding to the appearance of new stable states, Physica A 104, 480 (1980).

[88] M. Poggio, C. L. Degen, H. J. Mamin, and D. Rugar, Feedback Cooling of a Cantilever's Fundamental Mode Below $5 \mathrm{mK}$, Phys. Rev. Lett. 99, 017201 (2007).

[89] P. Huang, J. Zhou, L. Zhang, D. Hou, S. Lin, W. Deng, C. Meng, C. Duan, C. Ju, X. Zheng, Fei Xue, and J. Du, Generating giant and tunable nonlinearity in a macroscopic mechanical resonator from a single chemical bond, Nat. Commun. 7, 11517 (2016).

[90] G. Ranjit, M. Cunningham, K. Casey, and A. A. Geraci, Zeptonewton force sensing with nanospheres in an optical lattice, Phys. Rev. A 93, 053801 (2016).

[91] E. Hebestreit, M. Frimmer, R. Reimann, C. Dellago, F. Ricci, and L. Novotny, Calibration and energy measurement of optically levitated nanoparticle sensors, Rev. Sci. Instrum. 89, 033111 (2018).

[92] G. J. Feldman and R. D. Cousins, Unified approach to the classical statistical analysis of small signals, Phys. Rev. D 57, 3873 (1998). 